\title{
A non-intrusive reduced basis method for elastoplasticity problems in geotechnics
}

\author{
R. Chakir ${ }^{1}$ and J.K. Hammond ${ }^{1}$ \\ mail: rachida.chakir@ifsttar.fr; janelle.hammond@ifsttar.fr
}

\begin{abstract}
This work aims at investigating the use of reduced basis (RB) methods to diminish the cost of the resolution of parameter-dependent partial differential equations (PDEs) present in elastoplasticity problems arising from geotechnics modeling. Computation times for large three-dimensional analysis commonly take tens of hours, making optimization procedures or sensitivity analysis, relying on repeated simulations, hardly feasible. In many cases the analysis requires very specific features such as highly non-linear constitutive laws, involving a complex description of hardening phenomena in soils, which could not be solved using a standard reduced basis method. Given this constraint, an approach making it possible to use the reduced basis framework with any finite element software (without modifying the code) and considering it as a "black box" gives the so-called non-intrusive reduced basis method a versatility of great practical interest. Our approach involves the computation of less expensive (but less accurate) FE approximation during the online stage and improvement of those solutions using a RB-based rectification method. The chosen application belongs to the field of tunnel engineering, the particular problem being the displacement of the soil around a shallow tunnel for varying values of parameters: Young's modulus, friction angle, cohesion coefficient (characterizing the soil) and confinement loss (caused by the excavation of the tunnel). CESAR-LCPC, a FEM-based software, was used as a black-box to compute displacements during the offline and the online stages, whereas Freefem ++ was used for the implementation of the RB-based rectification method and analysis of the results. We proposed two rectification methods in the non-intrusive framework, and found that a modified rectification method was more adapted to the problem considered. With this non-intrusive method computation time has been reduced by $85 \%$ compared to $\mathbb{P}_{2}$ finite element method without loss of accuracy.
\end{abstract}

Keywords : Reduced Basis method; Finite Element method; Parametric studies; Elastoplasticity; Soils.

\section{Introduction}

Numerical modeling has met growing success over the last decades, becoming indispensible in the field of geotechnical engineering, leading to the resolution by finite elements of even larger nonlinear problems. This trend stems from the need to account for the influence of constructing new structures, such as deep foundations of high-rise buildings or shallow tunnels for transport infrastructures, on neighboring structures (e.g. sewers, existing buildings, etc.) in dense urban areas. Computation times for large three-dimensional analysis commonly take tens of hours, making sensitivity analysis relying on repeated simulations hardly feasible. A common approach is to develop simplified models, such as metamodels, to approximate the model without significant loss of accuracy. In [1] a metamodel

\footnotetext{
${ }^{1}$ Université Paris Est, IFSTTAR, 10-14 Bd Newton, Cité Descartes, 77447 Marne La Vallée Cedex, France.
} 
based on Proper Orthogonal Decomposition (POD) with radial basis functions (RBF) was applied to test problems in material mechanics with the goal of illustrating the capability of these metamodels to reproduce mechanical responses to the loading of complex non-linear material systems. An extended version of the POD-RBF metamodel was proposed in [2] to surrogate a 3D finite element simulation of a tunnel using Hardening Soil model.

Another approach to rapidly compute reliable approximations of solutions of complex problems with many parameters is reduced basis (RB) methods [3]. These methods rely on the parametric structure of the model and that when the parameters vary, the manifold of all possible solutions can be approximated by a low-dimensional space, the reduced basis space. The reduced basis is constructed from solutions of the parametrized problem for a well-chosen set of parameters. Standard reduced basis methods are Galerkin approximations of the full order model within a lower-dimensional reduced basis space. One of the keys of RB techniques is the decomposition of the computational work into offline and online stages. During the offline stage the reduced basis functions are computed, as well as all parameter-independent quantities. This is done only once, whereas parameter-dependent quantities are computed during the online stage. Application of the reduced basis method to linear elastic solid mechanics problems with parameters of different natures (either physical or geometrical) was proposed in $[4,5,6,7,8]$. The efficiency of the reduced basis or POD-based reduction methods relies on liberating online calculation costs from dependency on the discretization. However in elasto-plastic problems with highly nonlinear behavior, not uncommon in the field of soil mechanics, the computational complexity related to the local integration of the nonlinear constitutive laws is not reduced. Several alternative ways to carry out the standard POD-based reduction method for problems with nonlinear behavior were investigated. For example in $[9,10]$ a partial reduction is performed over the region of the domain with elastic behavior, while the plastified region remains unreduced. This selective POD-based model reduction was extented by an adaptive method of sub-structuring POD(A-SPOD) in which the subdomain where model reduction is applied is determined automatically. In $[11,12,13]$ a hyper-reduction approach was proposed by Ryckelynck to treat the problem of local dependency and extended by Zhang [14] to a thermo-elastoplastic model. The hyper reduction method consists in introducing reduced integration domains for internal variables.

However these methods require modification of the finite element calculation code leading to an intrusive procedure, which is particularly restrictive in the case of the considered geotechnics modeling applications. Analysis of the displacement field around a tunnel opening using numerical techniques is quite sensitive to the constitutive models of the soil used to described the fundamental behavior of the materials involved. In many cases the analysis requires very specific features which are not available in all finite element softwares, such as highly non-linear constitutive laws, involving a complex description of hardening phenomena in soils. Given this constraint, an approach making it possible to use reduced basis methods with any finite element software (without modifying the code) and considering it as a "black box" gives the so-called non-intrusive reduced basis method a versatility of great practical interest. Our approach involves the computation of less expensive FE approximation (but less accurate) with a black-box FE software and improvement of those solutions using a reduced basis during the online stage. In this work, we aim to demonstrate the feasibility of this approach - a two-grid finite-element/RB method, introduced in $[15,16]$ - to geotechnics modeling.

This paper is organized as follows. In Section 2, we formulate the elastoplastic problem providing a brief description of the physical system, the material behavior laws, the governing equations and boundary conditions. In Section 3, we provide a brief introduction to reduced basis methods and discuss a preliminary analysis of the feasibility and reliability of reduced basis approximations of the elastoplastic problem. In Section 4 the problem is solved with a non-intrusive reduced basis method. 
Finally Section 5 presents the conclusions.

\section{The elastoplastic problem}

The chosen application belongs to the field of tunnel engineering. In urban areas, it is necessary to consider the possible impact of the construction of a shallow tunnel on existing structures (buildings, foundations, etc.). In many cases, the first step consists of evaluating the settlements that would be induced by the construction of a tunnel in a "greenfield" environment, i.e. with no structure built at the surface. Let us consider a circular tunnel built through a $50-\mathrm{m}$ horizontal ground layer. The tunnel diameter is $\mathrm{D}=10 \mathrm{~m}$ and the axis depth is $\mathrm{H}=25 \mathrm{~m}$. The analysis is carried out under the plane strain assumption. If the ground is homogeneous and isotropic, only half of the ground layer needs to be considered. For practical reasons, the analysis is limited to a distance of $\mathrm{L}=100 \mathrm{~m}$ from the tunnel axis (see figure 1).

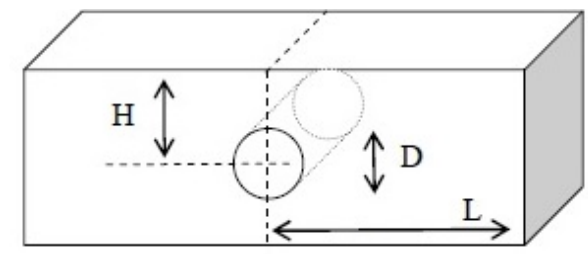

Figure 1: Geometry of the physical domain

\subsection{From material behavior laws to the governing equations}

In design calculations, materials (soil, concrete, rock, metal, liquid, gas) are considered as continuous mediums (or continua). These materials are thus considered to obey certain general physical and mechanical principles, such as the conservation of energy and momentum. Everyday experience can tell us that different materials do not behave in the same way under the same forces. General physics laws do not allow us to make the distinction between different sorts of materials. We therefore want to characterize the specific behavior of the continuum equivalent to the material under consideration. This is the goal of the constitutive laws associated to a material; the laws must characterize the evolution caused by given exterior forces and be specific to the material in question. When switching from one material to another, the laws must translate the differences in practically observed behavior. The constitutive law associated to a material is necessary to complete the system of equations of any mechanics problem of continua or structural design. The behavior of the soils in our problem is represented by an elastoplastic model used for pulverulent soils (sands) and for long-term coherent soils (clay and silt). Observations show that irreversible deformations appear when the stress exceeds a certain level. Let $u$ be the displacement vector; the deformation is assumed to be infinitesimal so that the strain tensor can be written as $\epsilon(u)=\frac{1}{2}\left(\nabla u+{ }^{t} \nabla u\right)$. The framework of plasticity is based on the assumption that the strains can be split into the sum of two terms :

$$
\epsilon=\epsilon^{e}+\epsilon^{p}
$$

where $\epsilon^{e}$ is the elastic strain tensor and $\epsilon^{p}$ is the plastic part of the total strain tensor $\epsilon$, which corresponds to the irreversible part of the strain. The elastic part of the behavior of the soil is linear ${ }^{2}$ and

\footnotetext{
${ }^{2}$ Let us note that here the term "linear" or "nonlinear" refers to the behavior of the material, not necessarily to a linear or nonlinear equation.
} 
isotropic and described by Hooke's law (characterized by Young's modulus $E$ and Poisson's coefficient $\nu)$. The plastic part of the soil's behavior is considered nonlinear and is obtained via the Mohr-Coulomb model [17] (characterized by the cohesion $c$, the friction angle $\varphi$, and the dilatancy angle $\psi$ ).

\subsubsection{Linear elastic behavior: Hooke's Law}

Hooke's law (2) describes the relationship between the stress tensor $\sigma(u) \in \mathbb{R}^{d \times d}$ and the elastic strain tensor $\epsilon^{e}(u) \in \mathbb{R}^{d \times d}$.

$$
\begin{aligned}
\sigma(u)-\sigma^{0}= & \frac{E \nu}{(1+\nu)(1-2 \nu)} \operatorname{tr}\left(\epsilon^{e}(u)\right) \mathbf{I}_{\mathbf{d}} \\
& +\frac{E}{(1+\nu)} \epsilon^{e}(u)
\end{aligned}
$$

with $\sigma^{0}$ the initial stress tensor, $E$ and $\nu$ soil's parameters.

\subsubsection{Nonlinear plastic behavior: Mohr Coulomb's model}

It is assumed that the plastic strain does not evolve as long as the stress tensor remains in the interior of a region of the stress space, called the elastic domain $[17,18]$. The elastic domain is generally defined by a condition of the type $f\left(\sigma_{i j}\right)<0$, where $f$ is called the yield function. The yield surface is the boundary of the elastic domain and thus defined by $f(\sigma)=0$. For sands, the yield function can be expressed as follows.

$$
f\left(\sigma_{i j}\right)=\left(\sigma_{\ell}-\sigma_{s}\right)-\left(\sigma_{s}+\sigma_{\ell}\right) \sin \varphi-2 c \cos \varphi,
$$

where $\sigma_{\ell}$ and $\sigma_{s}$ represent the largest and smallest eigenvalues of the stress tensor $\sigma$ (often call principal stresses in mechanics). The parameters $\varphi$ and $c$ are the friction angle and the cohesion characterizing the soil. In this study, we focus on the case of elastic-perfectly plastic models, in which the yield surface does not evolve with loading. Let us consider the stress tensor $\sigma_{i j}$ corresponding to a given load. If $f\left(\sigma_{i j}\right)<0$, then $\sigma_{i j}$ is in the elastic domain, and so we have that the deformation variation is described simply by

$$
d \epsilon=d \epsilon^{e} .
$$

If $f\left(\sigma_{i j}\right)=0$, then $\sigma_{i j}$ is on the boundary of the elastic domain. To describe the behavior at this point, we need to know if the material is in loading, in which case the deformation variation is described by

$$
d \epsilon=d \epsilon^{p}+d \epsilon^{e} .
$$

or if the material is unloading and has an elastic behavior. At a regular point $\sigma_{i j}$ of the elasticity boundary, the plastic deformation can be described by the so-called "plastic flow rule"

$$
d \epsilon^{p}=d \hat{\lambda} \frac{\partial g}{\partial \sigma}
$$

where $d \hat{\lambda} \geq 0$ is a scalar called the plastic multiplier and $g$ is given by

$$
g\left(\sigma_{i j}\right)=\left(\sigma_{\ell}-\sigma_{s}\right)-\left(\sigma_{s}+\sigma_{\ell}\right) \sin \psi-2 c \cos \psi .
$$

The problem is closed by the "consistency condition"

$$
d f d \hat{\lambda}=0 .
$$




\subsubsection{Equilibrium equation}

We consider a static process, then the equilibrium describing our system is:

$$
\operatorname{div}(\sigma)+\rho F=0
$$

where $\rho F=\left(\begin{array}{c}0 \\ -\gamma\end{array}\right)$ is the external body force and $\gamma$ is the volumetric weight of the soil.

The elastic deformation is linked to the variation of the stress by a linear relation:

$$
\sigma-\sigma^{0}=C: \epsilon^{e}
$$

From (2) one can see that $C_{i j k l}$ (representing the elasticity tensor of the material) is constant, symmetrical $\left(C_{i j k l}=C_{j i k l}=C_{i j l k}=C_{k l i j}\right)$ and depends only on $E$ and $\nu$.

\subsubsection{Boundary conditions}

In this paper, we consider a $2 \mathrm{D}$ problem on the bounded domain $\Omega \subset \mathbb{R}^{2}$ (see figures 2 and 3 ).
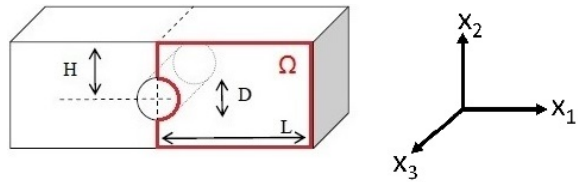

Figure 2: 2D representation of the domain $\Omega$

We will impose zero horizontal displacement on $\Gamma_{1}$ and $\Gamma_{3}$ and zero horizontal and vertical displacement on $\Gamma_{2}$. The load consists of a surface density of force $I$ applied on the wall of the tunnel $\left(\Gamma_{6}\right)$ calculated from the initial stress tensor (which we assumed geostatic):

$$
I=\lambda \sigma^{0} \cdot \vec{n}, \quad \text { with } \quad \sigma^{0}=\left(\begin{array}{cc}
x_{2} K_{0} \gamma & 0 \\
0 & x_{2} \gamma
\end{array}\right),
$$

where $\lambda$ represents the confinement loss caused by the excavation of the tunnel and $K_{0}=1-\sin (\varphi)$ the coefficient of the earth pressure at rest.

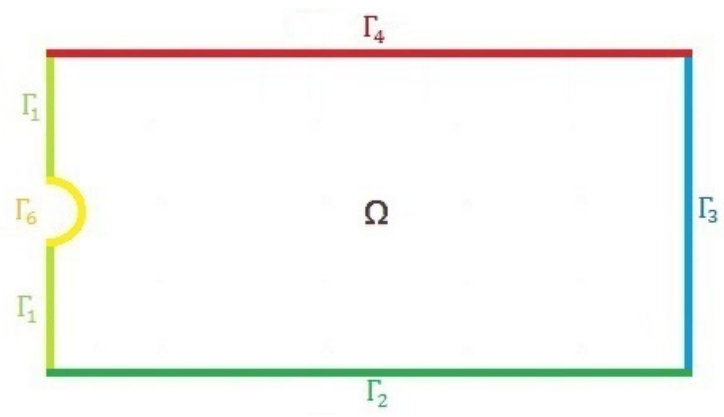

Figure 3: Boundaries of the domain $\Omega$ 
Let $\left(u_{i, i=1, \cdots, d}\right)$ be the displacement components in the $x_{i}$-directions in $\Omega$. The boundary conditions of our problem read as follows:

$$
\left\{\begin{array}{l}
\sigma \cdot \vec{n}=-\lambda \sigma^{0} \cdot \vec{n} \text { on } \Gamma_{6}, \\
u_{1}=0 \text { on } \Gamma_{1} \text { and } \Gamma_{3}, \\
(\sigma \cdot \vec{n})_{2}=0 \text { on } \Gamma_{1} \text { and } \Gamma_{3} \\
u_{i, i=1,2}=0, \text { on } \Gamma_{2}, \\
\sigma \cdot \vec{n}=\overrightarrow{0} \text { on } \Gamma_{4}
\end{array}\right.
$$

\subsection{Weak formulation and finite element approximation}

Associated to the physical domain $\Omega$, we defined the following functional space

$$
X=\left\{\begin{array}{l|l}
v \in\left(H^{1}(\Omega)\right)^{2} & \begin{array}{l}
v_{1}=0 \text { on } \Gamma_{1}, \Gamma_{2}, \Gamma_{3} \\
v_{2}=0 \text { on } \Gamma_{2}
\end{array}
\end{array}\right\}
$$

We then introduced the weak form of our elastoplastic problem, arising from (6) and (8):

Find $u \in X$ such that,

$$
\begin{aligned}
\int_{\Omega}\left(\epsilon(u)-\epsilon^{p}(u)\right): C: \epsilon(v) d \Omega & \\
= & \int_{\Omega} \rho F v d \Omega-\int_{\Omega} \sigma^{0}: \epsilon(v) d \Omega \\
& \quad-\int_{\Gamma_{6}} \sigma^{0} \vec{n} \cdot v d \Gamma, \quad \forall v \in X
\end{aligned}
$$

with $\epsilon_{i j}(u)=\frac{1}{2}\left(\frac{\partial u_{i}}{\partial x_{j}}+\frac{\partial u_{j}}{\partial x_{i}}\right)$.

In what follows, we fix the Poisson coefficient at $\nu=0.3$, the volumetric weight of the soil at $\gamma=$ $20 \mathrm{kN} / \mathrm{m}^{3}$ and assume that the dilatancy angle is equal to the friction angle $(\psi=\varphi)$.

Let us denote by $\mu=(E, \lambda, \varphi, c)$ our parameter set and by $\mathcal{D} \subset \mathbb{R}^{4}$ our parameter domain. We will decompose the left hand side of equation (10) into a linear term:

$$
a_{e}(u(\mu), v ; \mu)=\int_{\Omega} \epsilon(u(\mu)): C(\mu): \epsilon(v) d \Omega
$$

and a nonlinear term

$$
a_{p}\left(\epsilon^{p}(u(\mu)), v ; \mu\right)=\int_{\Omega} \epsilon^{p}(u(\mu)): C(\mu): \epsilon(v) d \Omega,
$$

and denote by $\mathcal{L}(v ; \mu)$ the right-hand side term

$$
\int_{\Omega} \rho F v d \Omega-\int_{\Omega} \sigma^{0}: \epsilon(v) d \Omega-\int_{\Gamma_{6}} \sigma^{0} \vec{n} \cdot v d \Gamma
$$

We consider a parametrized problem with varying values of $E \in[100 ; 300] M P a, \varphi \in[22 ; 34]$ degrees, $\lambda \in[0.2 ; 0.4]$ and $c \in[20 ; 40] k P a$ : for a given $\mu \in \mathcal{D}$, find $u(\mu) \in X$ such that, $\forall v \in X$,

$$
a_{e}(u(\mu), v ; \mu)-a_{p}\left(\epsilon^{p}(u(\mu)), v ; \mu\right)=\mathcal{L}(v ; \mu) .
$$

Let $\left\{\mathcal{T}_{h}\right\}_{h}$ be a family of regular triangulations of $\Omega$ and denote by $X_{h}$ the following $\mathbb{P}_{k}$ finite element space

$$
X_{h}=\left\{v=\left(v_{1}, v_{2}\right) \in X, \forall K \in \mathcal{T}_{h},\left.v_{i}\right|_{K} \in \mathbb{P}_{k}(K)\right\}
$$


The finite element discretization of (11) is as follows : for a given $\mu \in \mathcal{D}$, find $u_{h} \in X_{h}(\mu)$ such that,

$$
a_{e}\left(u_{h}(\mu), v_{h} ; \mu\right)-a_{p}\left(\epsilon^{p}\left(u_{h}(\mu)\right), v_{h} ; \mu\right)=\mathcal{L}\left(v_{h} ; \mu\right),
$$

In this work, CESAR-LCPC [19], a FEM-based software, has been used to solve (12), which employs the following iterative procedure (see algorithm 1 ) to approximate the displacement $u_{h}$, the stress tensor $\sigma_{h}$, the strain tensor $\epsilon_{h}$ and the plastic strain tensor $\epsilon_{h}^{p}$. For more details on the computational procedure see $[17,18]$.

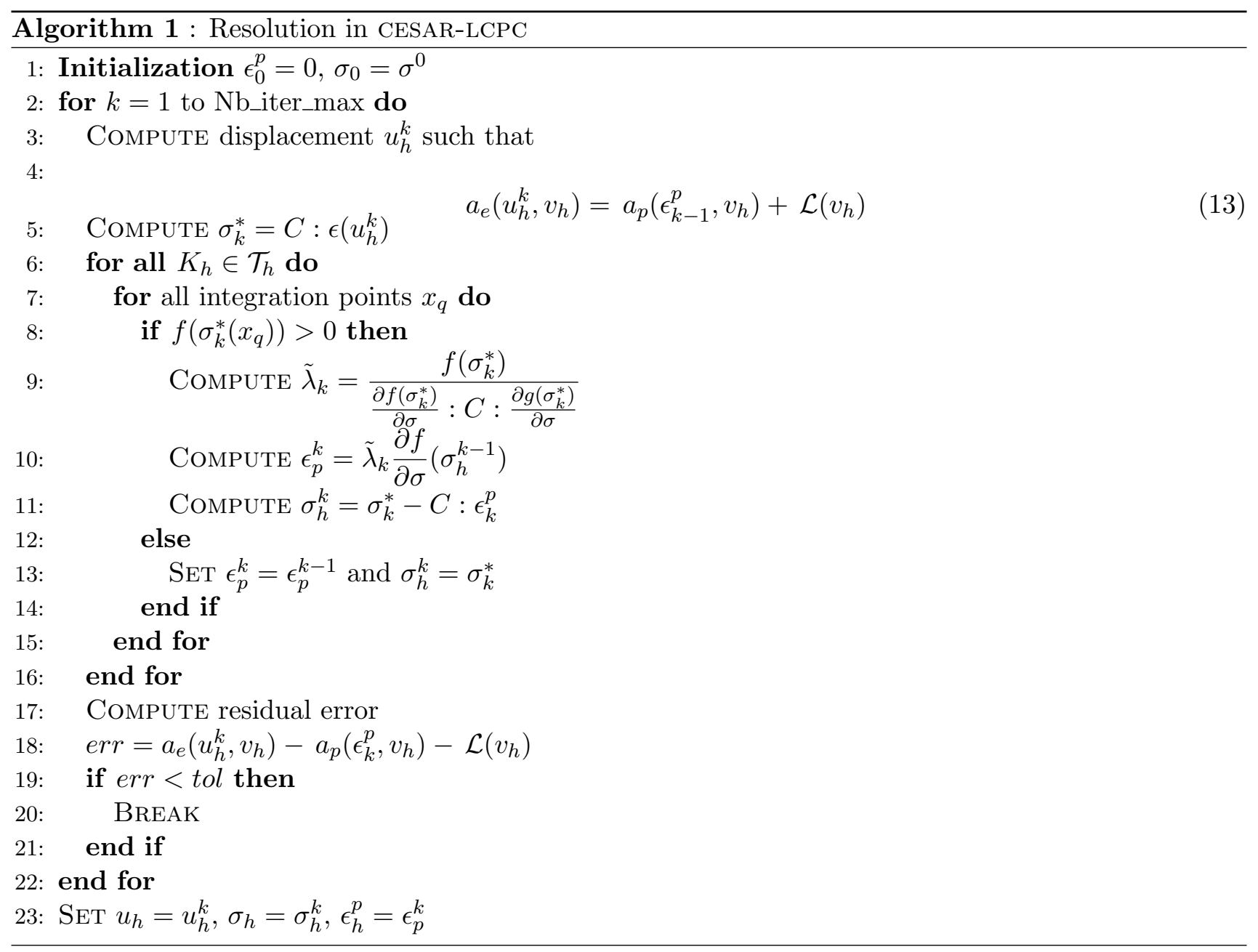

We want to apply reduced basis methods within this framework to compute the displacement $u_{h}$ corresponding to different values of $E, \lambda, \varphi, c$.

\section{Methodology}

The resolution of the problem introduced in the previous section can prove costly, particularly in the many-query context due to its parametric nature, making it an ideal candidate for reduced basis methods. The reduced basis method relies on the fact that when the parameters vary, the set of solutions is often of small Kolmogorov dimension, implying that $\mathcal{M}_{h}=\left\{u_{h}(\mu) \in X_{h} \mid \mu \in \mathcal{D}\right\}$, the manifold of all solutions, can be approximated by a finite set of well-chosen FE solutions of the parametrized PDE. 
One can identify a set of parameters, $S_{N}=\left(\mu_{1}, \mu_{2}, \cdots, \mu_{N}\right) \in \mathcal{D}^{N}$ such that the particular solutions $\left(u_{h}\left(\mu_{1}\right), \cdots, u_{h}\left(\mu_{N}\right)\right)$ will generate this low dimension space. The idea of reduced basis methods is to compute an inexpensive and accurate approximation, $u_{h}^{N}(\mu)$, of the solution to problem (11) for any $\mu \in \mathcal{D}$ by seeking a linear combination of the particular solutions $\left(u_{h}\left(\mu_{1}\right), \cdots, u_{h}\left(\mu_{N}\right)\right)$

$$
u_{h}^{N}(\mu)=\sum_{i=1}^{N} \alpha_{i}^{h}(\mu) u_{h}\left(\mu_{i}\right) .
$$

For a stable implementation of the reduced basis method, it is necessary to build a better basis than the one composed of the $\left\{u_{h}\left(\mu_{i}\right)\right\}_{1 \leq i \leq N}$, usually by a Gram-Schmidt method. In what follows, we denote by

$\left\{\xi_{1}, \cdots, \xi_{N}\right\}$ these $L^{2}$ orthonormalized basis functions, and by $X_{h}^{N}$ the approximation space which they engender: the reduced basis space. During the implementation of the reduced basis method, the computational work is separated into two stages: offline and online. This decomposition is a key ingredient of the method. The reduced basis functions, $\left\{\xi_{1}, \cdots, \xi_{N}\right\}$, as well as all expensive parameter-independent terms are computed once during the offline stage and stored, whereas during the online stage - for each new value of the parameters - inexpensive parameter-dependent quantities are evaluated, together with the computation of the coefficients $\alpha_{i}^{h}(\mu)$.

The usual RB method is a Galerkin method on the space $X_{h}^{N}$, which is of much smaller dimension than the original approximation space $X_{h}$, the resolution of the problem (13) in $X_{h}^{N}$ is less expensive than in the true finite element space $X_{h}$. However, to perform the online stage efficiently, one must isolate the parametric contribution to the corresponding linear system, allowing all parameter-independent matrices and vectors to be built only once and saved during the offline stage. In the case of Mohr-Coulomb's model used in CESAR-LCPC, a parameter-dependent term must be calculated at each integration point of the mesh (see Algorithm 1) during the iterative procedure implemented to solve (12); it is hence impossible to free the online stage of the FE complexity. This entirely nullifies the advantages of the RB method applied to our model. To overcome this flaw, we propose to use an alternative, less intrusive method, introduced in [16, 15], where coarse FE approximations are computed during the online stage, then projected into the reduced basis space and improved by a rectification technique.

We will begin by considering an analysis of the feasibility of RB methods for our problem (section 3.1 ), and will then discuss the non-intrusive method in more detail in section 3.2.

\subsection{POD analysis}

In order to determine if model reduction approaches, such as reduced basis methods or proper orthogonal decomposition (POD), can be applied to this problem, we will try to evaluate the complexity of the manifold $\mathcal{M}_{h}$ of all possible solutions induced by varying parameters. This analysis consists in a singular value decomposition method applied to the correlation matrix of solutions of (12) computed for different values of the parameters. Once the rapid decay rate of the singular values is confirmed, one can assume that RB method is worth implementing. Using CESAR-LCPC to compute $\mathbb{P}_{1}$ and $\mathbb{P}_{2}$-FE solutions of (12) for varying values of $\mu \in \Xi_{\text {test }}$ - a parameter set with sample size of $N_{\text {test }}=525$ selected over the parameter domain $\mathcal{D}$ - a correlation matrix of $L^{2}$-norm scalar products $\left(u_{h}\left(\mu_{i}\right), u_{h}\left(\mu_{j}\right)\right)_{L^{2}(\Omega), 1 \leq i, j \leq N_{\text {test }}}$ was computed. An $L^{2}$-orthonormalized POD basis was constructed using the following eigenfunctions

$$
w_{k}=\frac{1}{\sqrt{\lambda_{k}}} \sum_{\ell=1}^{N_{t e s t}} v_{k}(\ell) u_{h}\left(\mu_{\ell}\right) \quad 1 \leq k \leq N_{\text {test }},
$$

where $v_{k}(\ell)$ represents the $\ell^{t h}$ component of the $k^{\text {th }}$ eigenvector of the correlation matrix when ordered by decreasing eigenvalues. 
Let $P_{k}^{P O D}$ be the $L^{2}$-orthogonal projection operator from $X_{h}$ into the space $X_{h}^{k, P O D}$, spanned by the $k$ first POD basis functions $w_{k}$. Each test solution $\left\{u_{h}\left(\mu_{i}\right), \mu_{i} \in \Xi_{\text {test }}\right\}$ was projected onto $X_{h}^{k, P O D}$ to analyze the ability of the POD basis to approach the manifold $\mathcal{M}_{h}$, depending on the number of POD modes. In figure 4 we can see the associated errors plotted along with the eigenvalues of the matrix, where the average error is

$$
\frac{1}{N_{\text {test }}} \sum_{i=1}^{N_{\text {test }}}\left\|u_{h}\left(\mu_{i}\right)-P_{k}^{P O D} u_{h}\left(\mu_{i}\right)\right\|_{L^{2}}
$$

and the maximal error is

$$
\left\|u_{h}\left(\mu_{\max }^{k}\right)-P_{k}^{P O D} u_{h}\left(\mu_{\max }^{k}\right)\right\|_{L^{2}}
$$

with

$$
\mu_{\text {max }}^{k}=\underset{\mu_{i} \in \Xi_{\text {test }}}{\operatorname{argmax}}\left\|u_{h}\left(\mu_{i}\right)-P_{k}^{P O D} u_{h}\left(\mu_{i}\right)\right\|_{L^{2}}
$$
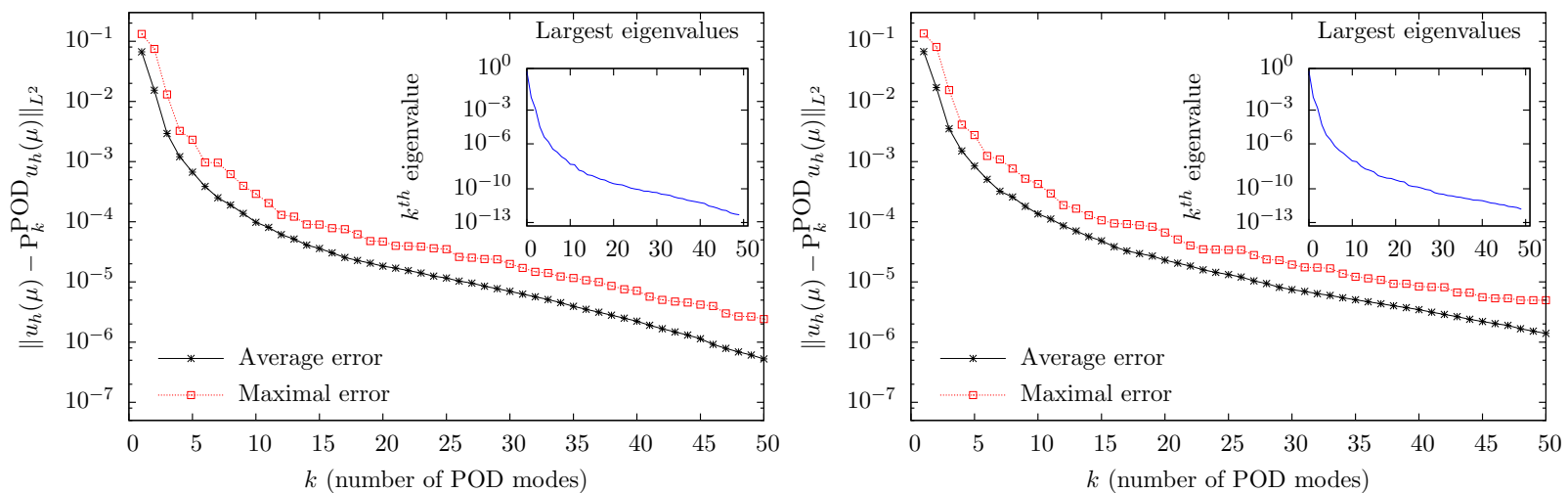

Figure 4: Relative errors of the POD projection with $\mathbb{P}_{1}$-FE (left) and $\mathbb{P}_{2}$-FE (right) snapshots.

We observe that the eigenvalues decay rapidly and that the projection errors are quite small. Figure 5 shows the average POD projection errors with respect to so-called reference solutions, computed on a very fine mesh for parameter values not included in the sample space solutions. These errors are plotted with the fine FEM error for comparison. Figure 6 displays the corresponding maximal errors.
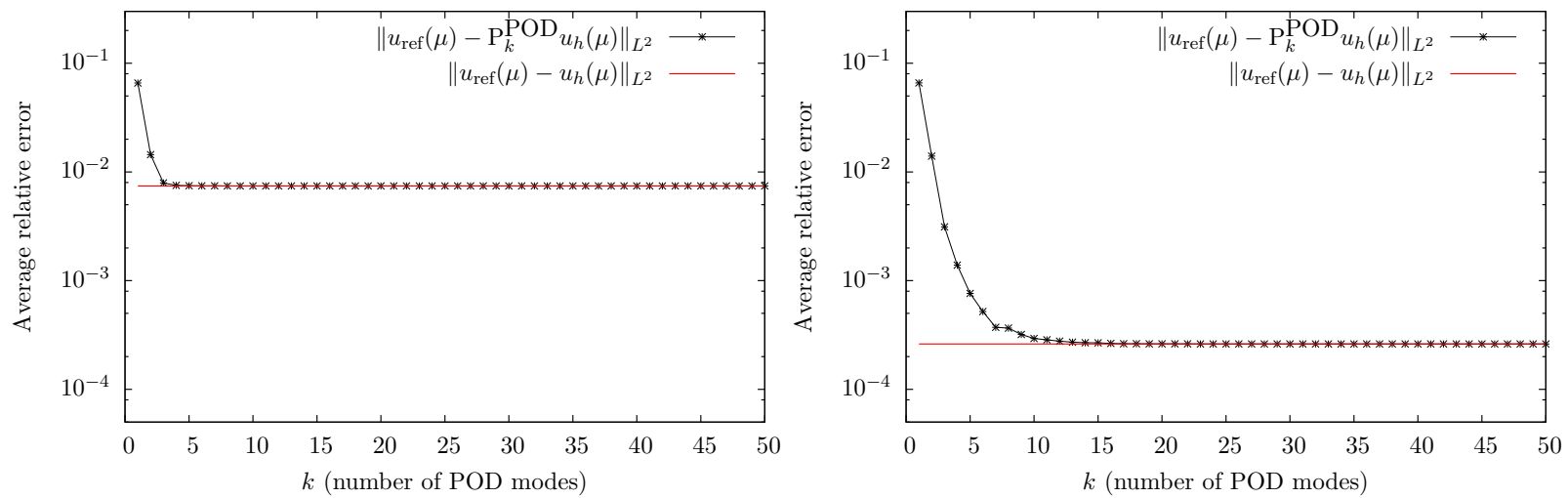

Figure 5: Average error of the POD projection vs FEM error using $\mathbb{P}_{1}$-FE (left) and $\mathbb{P}_{2}$-FE (right) snapshots 

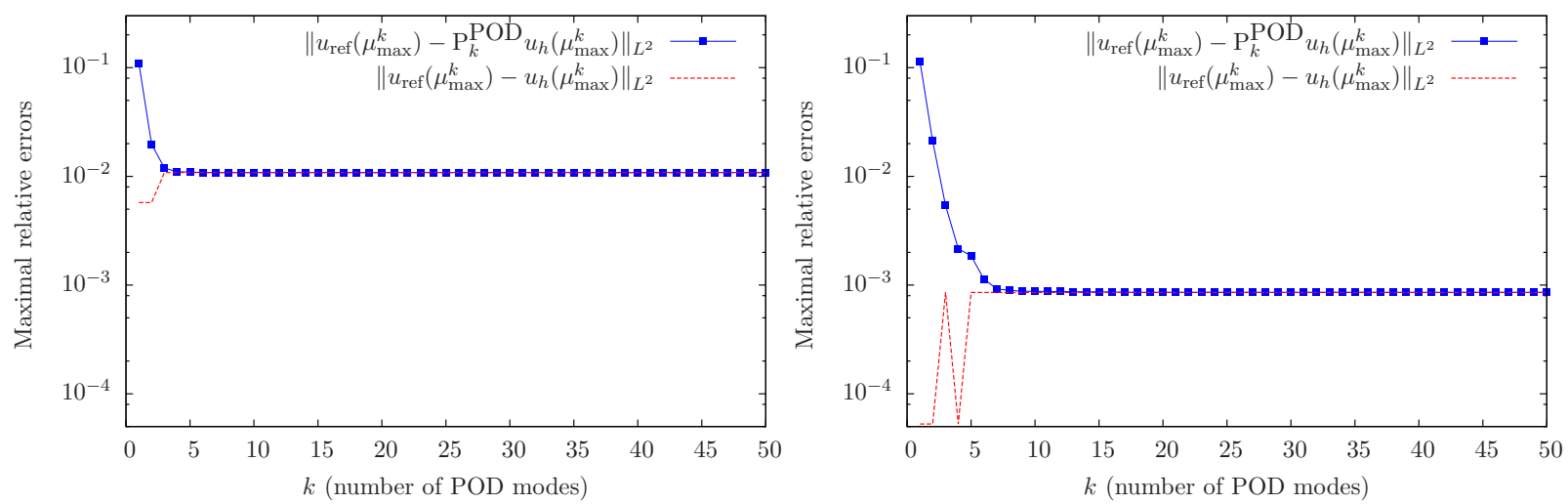

Figure 6: Maximal error of the POD projection vs FEM error using $\mathbb{P}_{1}$ (left) and $\mathbb{P}_{2}$ (right) snapshots

We can see that with only $k=5$ POD modes, the POD projection errors reach the same level of accuracy as the $\mathbb{P}_{1}$ FEM errors. As for the $\mathbb{P}_{2}$ FE errors, we only need about $k=10$ POD nodes. This suggests that a reduced basis approach is worth implementing.

\subsection{A non-intrusive reduced basis method: two-grid $F E / R B$ method with a rectification approach}

A popular strategy for constructing a reduced basis in the case of parameter-dependent problems is to use greedy algorithms, based on the idea of selecting the locally optimal element at each step. This option can be seen as an alternative to the POD strategy of the previous section. If we have an appropriate a priori error estimator to avoid full resolution of the problem to compute the test solutions, the greedy algorithm can be very low-cost. Knowing the Kolmogorov dimension of the solution space is relatively small, we can fix a maximum number $N_{g}$ of basis functions to be computed by the Greedy algorithm (given below, algorithm 2). Additionally, for stable implementation the chosen basis functions are $L^{2}$-orthonormalized with a Gram-Schmidt method.



Standard reduced basis methods are based on a Galerkin approach, whereas the two-grid FE/RB method involves the computation of a less expensive FE solution and improvement of this solution using the reduced basis. The standard reduced basis method aims at evaluating the coefficients $\alpha_{i}^{h}(\mu)$ intervening in the decomposition (14) of $u_{h}^{N}(\mu)$, which can appear as a substitute to the optimal coefficients

$$
\beta_{i}^{h}(\mu)=\left(u_{h}(\mu), \xi_{i}\right)_{L^{2}}
$$


corresponding to the decomposition of the $L^{2}$-projection of $u_{h}(\mu)$ into the space $X_{h}^{N}$. Let $\left\{\mathcal{T}_{H}\right\}_{H}$ be a family of "coarse" regular triangulations of $\Omega$, such that $H>>h$; we denoted by $X_{H}$ the coarse FE approximation space associated to this mesh, and by $u_{H}(\mu)$ the coarse FE approximation of (12) on $X_{H}$. The alternative two-grid $\mathrm{FE} / \mathrm{RB}$ method consists in proposing another surrogate to the coefficients $\beta_{i}^{h}(\mu)$ defined by

$$
\beta_{i}^{H}(\mu)=\left(u_{H}(\mu), \xi_{i}\right)_{L^{2}},
$$

While coarse FE approximations can be computed quickly enough to be used in the online stage, they may not be accurate enough for practical use. As the computation of $u_{H}(\mu)$, for $H>>h$, is significantly less expensive than that of $u_{h}(\mu)$, with the mesh size $H$ (chosen adequately) the coefficients $\beta_{i}^{H}(\mu)$ can be used to compute a low-dimensional approximation :

$$
\sum_{i=1}^{N} \beta_{i}^{H}(\mu) \xi_{i}
$$

Let $P_{N}^{H}$ be the $L^{2}$-projection operator from $X_{H}$ into the space $X_{h}^{N}$. Considering that we have used embedded FE spaces, namely $X_{H} \subset X_{h}$, we have $P_{N} u_{H}(\mu)=P_{N}^{H} u_{H}(\mu)$, and in consequence we will simplify the notation by also denoting by $P_{N}$ the $L^{2}$ projection operator $P_{N}^{H}$.

To improve even further the accuracy of this technique we propose to perform a rectification of the $P_{N} u_{H}(\mu)$. This is so far an empirical approach, which leads to great improvements in practice. A first explanation of the successful post-processing strategy first presented in [15] and then used in [20] in the framework of reduced basis simulation of PDE's can be found in [21]. This treatment will ensure that for the parameters $\left\{\mu_{i}\right\}_{1 \leq i \leq N}$ used in the construction of the reduced basis, the method returns exactly $\beta_{i}^{h}\left(\mu_{i}\right)$. In practice, we want to identify a so-called rectification matrix $R^{N}$ associated to the transformation $\mathcal{R}_{N}$ such that :

$$
\mathcal{R}_{N} P_{N} u_{H}\left(\mu_{i}\right)=P_{N} u_{h}\left(\mu_{i}\right) \quad \forall 1 \leq i \leq N .
$$

Since $\left\{\beta_{j}^{h}\left(\mu_{i}\right)\right\}_{1 \leq j \leq N}$ and $\left\{\beta_{j}^{H}\left(\mu_{i}\right)\right\}_{1 \leq j \leq N}$ are the optimal coefficients intervening in the decomposition of $P_{N} u_{h}\left(\mu_{i}\right)$ and $P_{N} u_{H}\left(\mu_{i}\right)$, the standard matrix, denoted by $A_{N}$, associated to the transformation $\mathcal{R}_{N}$ is equal to

$$
A_{N}=\left(\mathbf{B}_{h}^{N}\right) \times\left(\mathbf{B}_{H}^{N}\right)^{-1} \text { with } A_{N} \in \mathbb{R}^{N \times N},
$$

where $\mathbf{B}_{h}^{N}=\left(\begin{array}{c|c|c}\beta_{1}^{h}\left(\mu_{1}\right) & \cdots & \beta_{1}^{h}\left(\mu_{N}\right) \\ \vdots & \vdots & \vdots \\ \beta_{N}^{h}\left(\mu_{1}\right) & \cdots & \beta_{N}^{h}\left(\mu_{N}\right)\end{array}\right)$ and $\mathbf{B}_{H}^{N}=\left(\begin{array}{c|c|c}\beta_{1}^{H}\left(\mu_{1}\right) & \cdots & \beta_{1}^{H}\left(\mu_{N}\right) \\ \vdots & \vdots & \vdots \\ \beta_{N}^{H}\left(\mu_{1}\right) & \cdots & \beta_{N}^{H}\left(\mu_{N}\right)\end{array}\right)$.

Let us note that, contrarily to the $u_{h}(\mu)$, which we don't want to compute for a large number of values of $\mu$, the true solutions $u_{h}\left(\mu_{i}\right)$ have already been computed to build the reduced basis, making the computation of $A_{N}$ relatively cheap. For each new value of $\mu$, the coefficients $\beta_{i}^{H}(\mu)$ will be replaced by $\sum_{k=1}^{N} A_{i k}^{N} \beta_{k}^{H}(\mu)$, and an improved two-grid FE/RB approximation to equation (12), for $R_{N}=A_{N}$, can be :

$$
\mathcal{R}_{N} P_{N} u_{H}(\mu)=\sum_{i, j=1}^{N} A_{i j}^{N} \beta_{j}^{H}(\mu) \xi_{i}^{N}
$$

In our problem, we noticed that $A_{N}$ was rather poorly conditioned, and propose here a pre-processing to improve the rectification. Instead of computing the coefficients from the fine and coarse RB solutions, 
we will consider the previously computed POD basis functions to construct another rectification matrix $K_{N}$. To do so, in addition to the POD basis function $w_{k}$ introduced in the previous section, we introduced "coarse" POD basis function

$$
w_{k}^{H}=\sum_{\ell=1}^{N_{\text {test }}} v_{k}(\ell) u_{H}\left(\mu_{\ell}\right) \quad 1 \leq k \leq N .
$$

We defined a pre-processing matrix

$$
\begin{aligned}
& D_{N}=\left(\mathbf{F}_{h}^{N}\right) \times\left(\mathbf{F}_{H}^{N}\right)^{-1}, \\
& \text { where } \mathbf{F}_{h}^{N}=\left(\begin{array}{c|c|c}
\left(w_{1}, \xi_{1}\right)_{L^{2}} & \cdots & \left(w_{N}, \xi_{1}\right)_{L^{2}} \\
\vdots & \vdots & \vdots \\
\left(w_{1}, \xi_{N}\right)_{L^{2}} & \cdots & \left(w_{N}, \xi_{N}\right)_{L^{2}}
\end{array}\right) \text { and } \mathbf{F}_{H}^{N}=\left(\begin{array}{c|c|c}
\left(w_{1}^{H}, \xi_{1}\right)_{L^{2}} & \cdots & \left(w_{N}^{H}, \xi_{1}\right)_{L^{2}} \\
\vdots & \vdots & \vdots \\
\left(w_{1}^{H}, \xi_{N}\right)_{L^{2}} & \cdots & \left(w_{N}^{H}, \xi_{N}\right)_{L^{2}}
\end{array}\right) \text {. }
\end{aligned}
$$

We then construct the new rectification matrix $K_{N}$ as follows, for a suitable $N_{\max }$.

$$
K_{N}=\left(\begin{array}{cc}
D_{N_{\max }} & 0 \\
0 & T_{N}
\end{array}\right)
$$

with $T_{N}=\frac{1}{N}\left(\begin{array}{ccc}1 & & 0 \\ & \ddots & \\ 0 & & 1\end{array}\right) \in \mathbb{R}^{\left(N-N_{\max }\right) \times\left(N-N_{\max }\right)}$. By "cutting off" the chosen rectification before significant increases in the condition number (at $N_{\max }$ ), we can prevent associated peaks in error, thus achieving the results of $K_{N}$. Figure 7 shows condition numbers for the three proposed matrices: $A_{N}$, $D_{N}$, and $K_{N}$. Figure 8 shows rectification errors for the three proposed rectification matrices. We can see that the matrix $D_{N}$ is better conditioned than the matrix $A_{N}$ and that the rectification process is improved. However the most significant improvements are seen with matrix $K_{N}$.

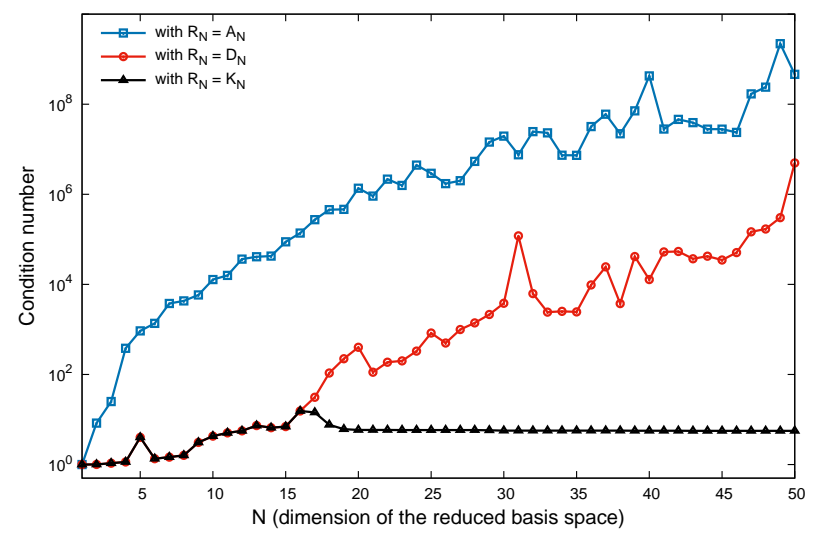

Figure 7: Condition number of the different rectification matrices: $A_{N}, D_{N}$, and $K_{N}$ during the offline stage $\left(\mathbb{P}_{2}\right.$ FEM solutions) 


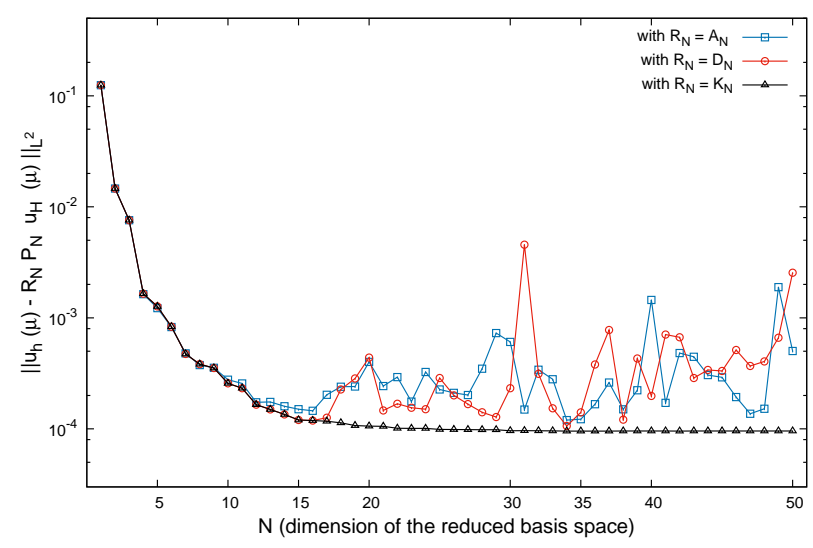

Figure 8: Average rectification errors the offline stage $\left(\mathbb{P}_{2}\right.$ FEM solutions) depending on the rectification matrix

\section{Numerical experiments}

The above-described method was applied to the problem using CESAR-LCPC for the FE resolution of equation (12) and FreeFem $++[22]$ was used for the implementation of the two-grid FE/RB method and analysis of the results.

Three meshes were considered: a coarse mesh $\mathcal{T}_{H}$ for the inexpensive computation of coarse solutions $u_{H}(\mu)$, a fine mesh $\mathcal{T}_{h}$ for the computation of satisfactory solutions used in the construction of the reduced basis, and a reference mesh $\mathcal{T}_{\text {ref }}$ considered fine enough to provide true solutions used for error calculation. See figure 9 below.

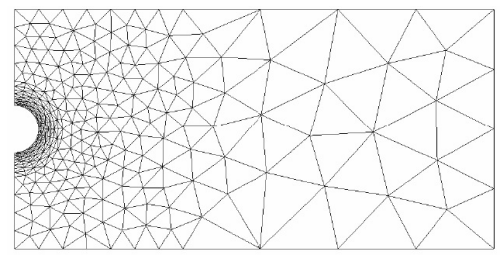

$\mathbb{P}_{2}$ ndof $=1247$

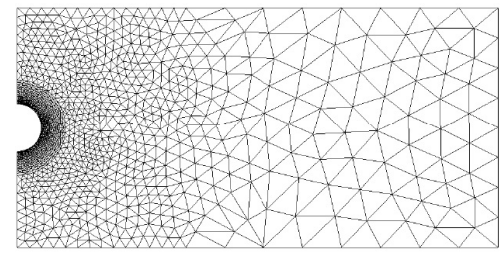

$\mathbb{P}_{2}$ ndof $=4853$

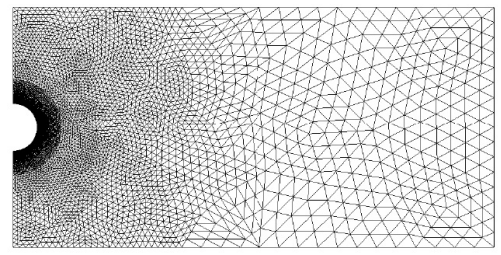

$\mathbb{P}_{2}$ ndof $=19143$

Figure 9: Coarse (left), fine (middle) and reference (right) embedded meshes used to compute FEM solutions

A parameter set $\Xi_{\text {trial }}$ with sample size of $N_{\text {trial }}=16$ is selected over $\mathcal{D} \backslash \Xi_{\text {test }}$ to test our method with $\mathbb{P}_{2}$ FEM grids. While in some applications, the simple rectification with $R_{N}=A_{N}$ will achieve the desired results, in this case the significant variation between coarse and fine solutions used to build the rectification matrix caused inadequate rectification results. We thus used matrix $R_{N}=K_{N}$ introduced in the previous section to improve the rectification. Figure 10 shows rectification errors during the offline stage. In figure 11, we can see the two-grid reduced basis method errors using rectification matrix $R_{N}=K_{N}$, for $N=16$; the error reaches the same order of precision as the $\mathbb{P}_{2}$-FEM fine solutions. We note that while rectification error in figure 10 does not descend further for $N \geq N_{\max }$, in contrast to the fine projection errors during the offline stage, figure 11 shows that the rectification approximation online does attain the same precision as the fine FEM solution. Figure 12 shows the actual displacement for a given parameter, $\mu=\mu_{\max }=\underset{\mu \in \Xi_{\text {trial }}}{\operatorname{argmax}}\left\|u_{h}(\mu)-R_{N} P_{N} u_{H}(\mu)\right\|_{L^{2}}=(125,0.35,23,0.03)$. The application of this problem being to evaluate impact on surface structures, we can consider displacement at the surface to be a quantity of interest. 

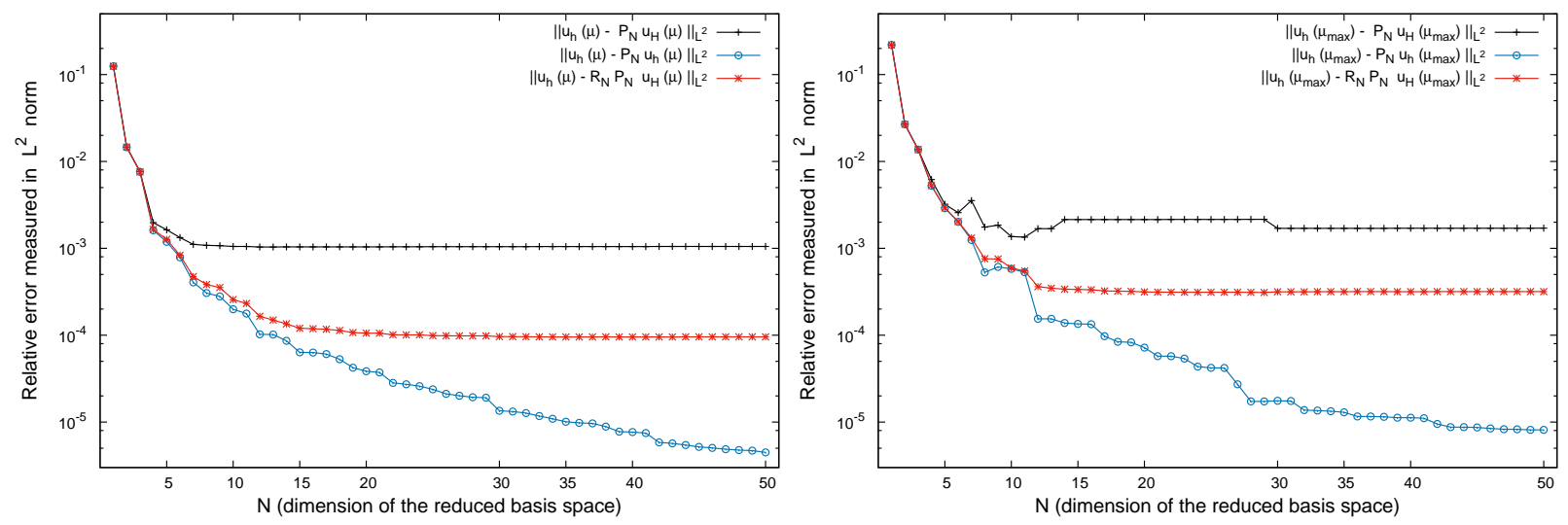

Figure 10: Average (left) and maximal (right) rectified RB projection errors on test space during the offline stage with $R_{N}=K_{N}$ and $N_{\max }=16$
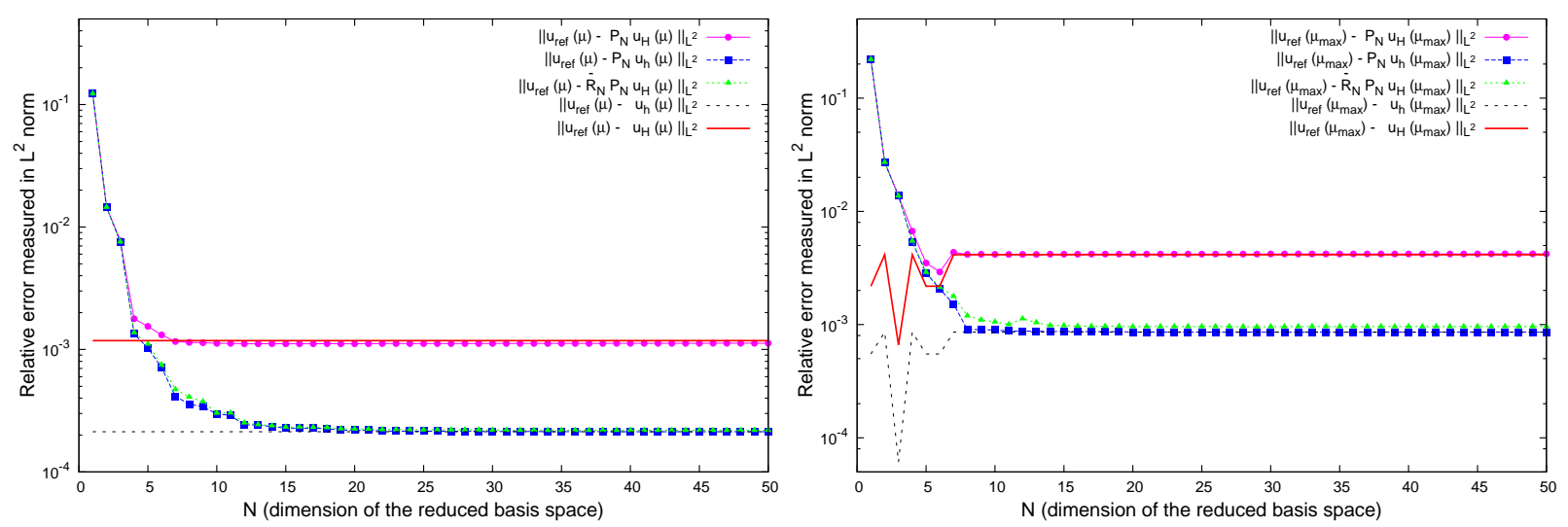

Figure 11: Average (left) and maximal (right) rectified RB projection errors on trial space during the online stage with $R_{N}=K_{N}$ and $N_{\max }=16$
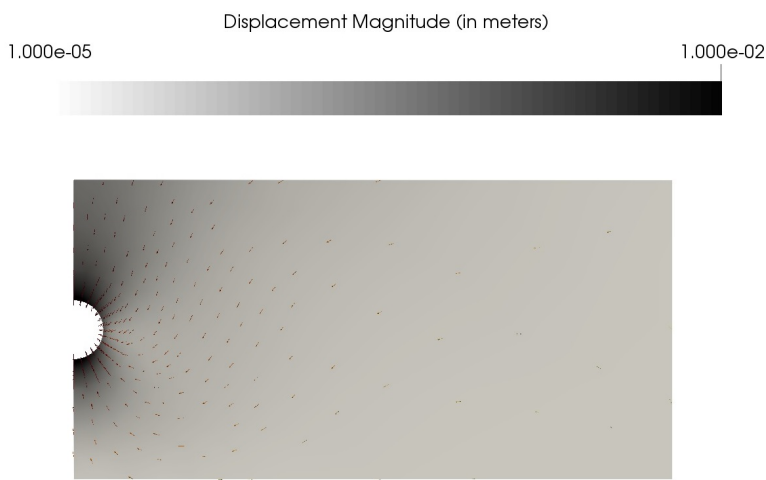

Figure 12: Displacement value for $\mu_{\max }=(125,0.35,23,0.03)$

Figure 13 shows error maps with respect to the $\mathbb{P}_{2}$-FE approximation over the calculation domain at various $N$-values of the two-grid $\mathrm{FE} / \mathrm{RB}$ method with and without the rectification, where the parameter value $\mu_{\max }=(125,0.35,23,0.03)$ corresponds to the solution with maximal error. We can see 
that the errors of the rectified solution with respect to the non rectified solution. Figure 14 shows errors over the calculation domain for $N=15$ with respect to the very fine reference solution, again for $\mu_{\max }=(125,0.35,23,0.03)$. We can see that the rectified solution errors closely resemble the fine FEM errors.



$\mathrm{N}=1$

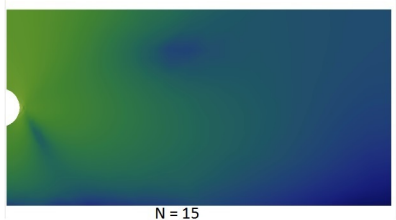

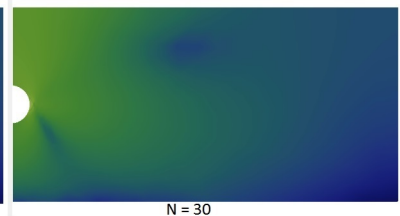

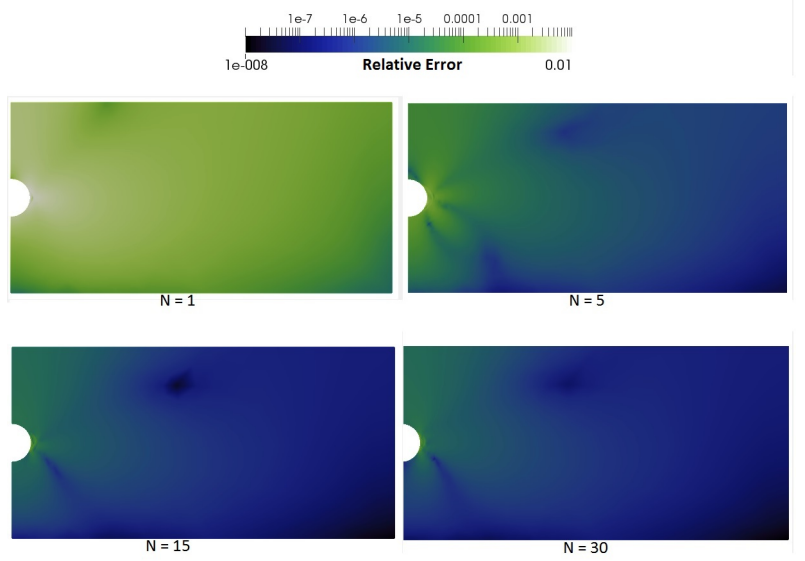

Figure 13: Relative error maps of the two-grid FE/RB approximation without (left) and with (right) rectification as function of $\mathrm{N}$ for $\mu=\mu_{\max }=(125,0.35,23,0.03)$

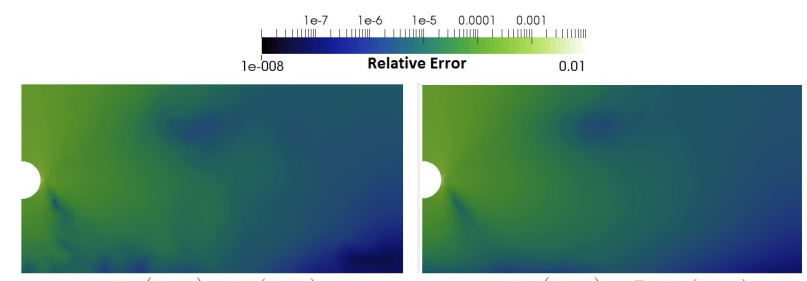

$u_{\operatorname{ref}}\left(\mu_{\max }\right)-u_{H}\left(\mu_{\max }\right)$ $u_{\mathrm{ref}}\left(\mu_{\max }\right)-P_{N} u_{H}\left(\mu_{\max }\right)$

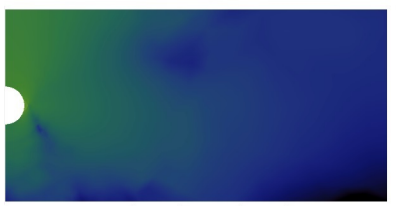

$u_{\mathrm{ref}}\left(\mu_{\max }\right)-u_{h}\left(\mu_{\max }\right)$

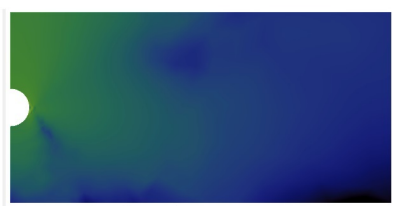

$u_{\text {ref }}\left(\mu_{\max }\right)-P_{N} u_{H}\left(\mu_{\max }\right)$

Figure 14: Error maps for $\mathrm{N}=15$ and $\mu_{\max }=(125,0.35,23,0.03)$

In figure 15 we can see a plot of the vertical displacement of the surface soil as a function of distance from the tunnel. The most significant displacement occurs, of course, nearest the tunnel. 


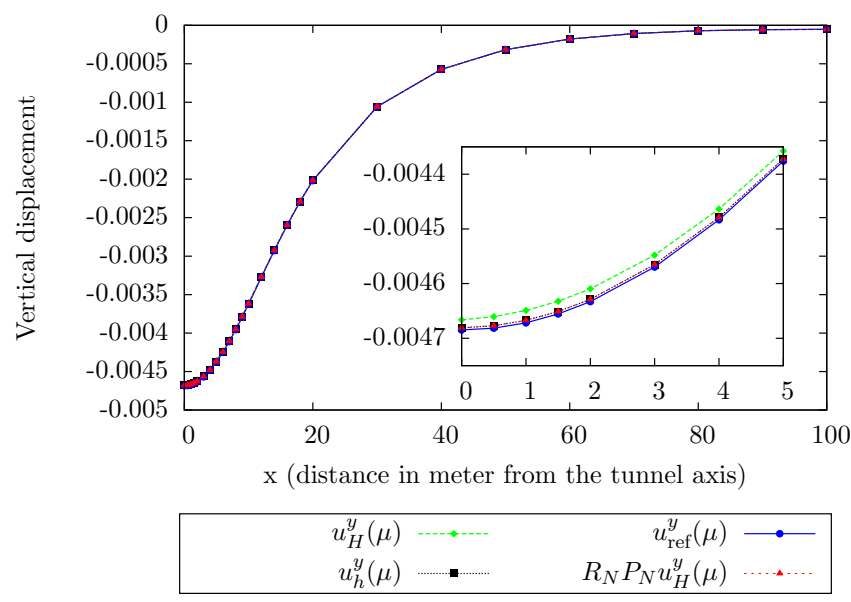

Figure 15: Value of the vertical displacement on the surface for $\mu=\mu_{\max }=(125,0.35,23,0.03)$

Figure 1 and table 16 show computation times for finite element simulations and the proposed online reduced basis method. We can see that satisfactory results can be obtained in a total of $3.17 \mathrm{~s}$ over the full domain, a reduction by $85 \%$ of computation time compared to a fine finite element approximation. In the case of many-query approximations - such as parametric studies, and possibly optimization procedures which are currently too computationally expensive for practical use - this reduction would prove to be significant.

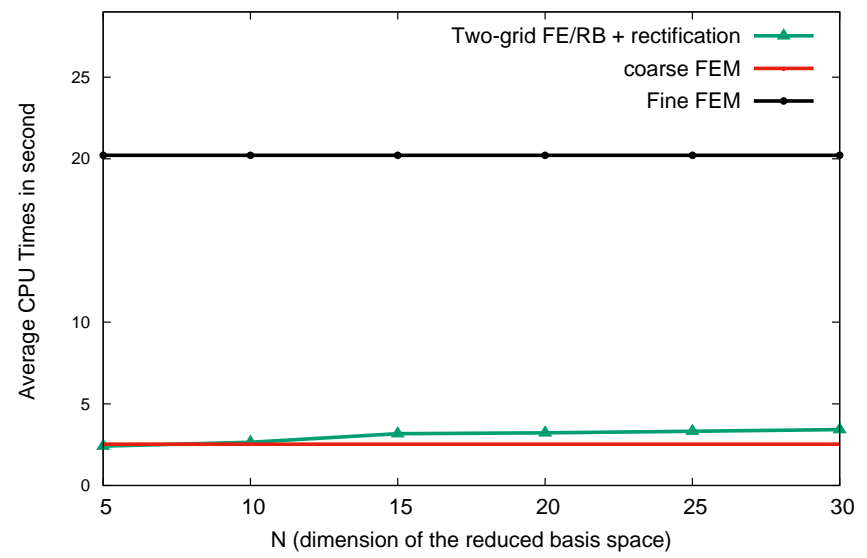

Figure 16: Comparaison of calculation times of $\mathbb{P}_{2}$-FE and two-grid FE/RB Methods

\begin{tabular}{|c|c|}
\cline { 2 - 2 } \multicolumn{1}{c|}{} & CPU Times \\
\hline Coarse FEM & $2.41 \mathrm{~s}$ \\
\hline Two-Grid RB/FE & $3.17 \mathrm{~s}$ \\
\hline Fine FEM & $20.22 \mathrm{~s}$ \\
\hline
\end{tabular}

Table 1: Comparaison of calculation times of $\mathbb{P}_{2}-\mathrm{FE}$ and two-grid $\mathrm{FE} / \mathrm{RB}$ Methods (for $\mathrm{N}=15$ ) 


\section{Conclusions}

In this paper we proposed a non-intrusive reduced basis method for application to the parametrized PDEs governing an elastoplasticity problem which could not be solved using a standard reduced basis method. We demonstrated the small dimension of the solution space affiliated to the problem using POD analysis. We then proposed two rectification methods in the non-intrusive framework, and found that a modified rectification method was more adapted to the problem considered. The particular problem being the displacement of the soil around a shallow tunnel, the displacement at the surface approximated by the reduced model was considered, showing the successful approximation results held true when considering only the most important area of the domain.

The results of this study demonstrate the feasibility of the presented two-grid non-intrusive reduced basis method in geotechnics modeling, a domain for which reduced modeling techniques can provide great benefit. Specifically, this technique is well-adapted to the particular PDE problem studied considering its non-intrusive nature.

\section{Acknowledgment}

The authors would like to thank Emmanuel Bourgeois for fruitful discussions and very valuable advice regarding our numerical simulations with CESAR-LCPC.

This research did not receive any specific grant from funding agencies in the public, commercial or not-for-profit sectors.

\section{References}

[1] Gabriella Bolzon and Vladimir Buljak. An effective computational tool for parametric studies and identification problems in materials mechanics. Comput Mech, 48(6):675-687, 2011.

[2] K. Khaledi and S. Miro. Robust and reliable metamodels for mechanized tunnel simulations. Computers and Geotechnics, 61:1-12, 2014.

[3] Christophe Prud'homme, Dimitrios V. Rovas, Karen Veroy, Luc Machiels, Yvon Maday, Anthony T. Patera, and Gabriel Turinici. Reliable real-time solution of parametrized partial differential equations: Reduced-basis output bound methods. Journal of Fluids Engineering, 124(1):70-80, 2002.

[4] K.C. Hoang, P. Kerfriden, and S.P.A. Bordas. A fast, certified and "tuning free" two-field reduced basis method for the metamodelling of affinely-parametrised elasticity problems. Computer Methods in Applied Mechanics and Engineering, 298:121-158, 2016.

[5] D. B. P. Huynh and A. T. Patera. Reduced basis approximation and a posteriori error estimation for stress intensity factors. International Journal for Numerical Methods in Engineering, 72(10):1219$1259,2007$.

[6] G. R. Liu, Khin Zaw, Y. Y. Wang, and B. Deng. A novel reduced-basis method with upper and lower bounds for real-time computation of linear elasticity problems. Computer Methods in Applied Mechanics and Engineering, 198(2):269-279, 2008.

[7] Roberto Milani, Alfio Quarteroni, and Gianluigi Rozza. Reduced basis method for linear elasticity problems with many parameters. Computer Methods in Applied Mechanics and Engineering, 197(51):4812-4829, 2008. 
[8] Karen Veroy. Reduced-basis methods applied to problems in elasticity: Analysis and applications. PhD thesis, Massachusetts Institute of Technology, 2003.

[9] M. Dossi A. Corigliano and S. Mariani. Model order reduction and domain decomposition strategies for the solution of the dynamic elastic-plastic structural problem. Computer Methods in Applied Mechanics and Engineering, 290:127-155, 2015.

[10] Annika Radermacher and Stefanie Reese. Model reduction in elastoplasticity: proper orthogonal decomposition combined with adaptive sub-structuring. Comput Mech, 54(3):677-687, April 2014.

[11] D. Ryckelynck. Hyper Reduction of finite strain elasto-plastic models. International Journal of Material Forming, 2(1):567-571, 2009.

[12] D. Ryckelynck and D. Missoum Benziane. Multi-level a priori hyper-reduction of mechanical models involving internal variables. Computer Methods in Applied Mechanics and Engineering, 199(1720):1134-1142, 2010.

[13] David Ryckelynck, Florence Vincent, and Sabine Cantournet. Multidimensional a priori hyperreduction of mechanical models involving internal variables. Computer Methods in Applied Mechanics and Engineering, 225-228:28-43, 2012.

[14] Yancheng Zhang, Alain Combescure, and Anthony Gravouil. Efficient hyper reduced-order model (HROM) for parametric studies of the 3d thermo-elasto-plastic calculation. Finite Elements in Analysis and Design, 102:37-51, 2015.

[15] Rachida Chakir and Yvon Maday. A two-grid finite-element/reduced basis scheme for the approximation of the solution of parameter dependent PDE. In Actes de congrès du 9ème colloque national en calcul des structures, Giens, 2009.

[16] Rachida Chakir and Yvon Maday. Une méthode combinée d'éléments finis à deux grilles/bases réduites pour l'approximation des solutions d'une EDP paramétrique. Comptes Rendus Mathematique, 347(7):435-440, 2009.

[17] Sophie Coquillay. Prise en compte de la non linéarité du comportement des sols soumis à de petites déformations pour le calcul des ouvrages géotechniques. PhD thesis, Ecole des Ponts ParisTech, 2005.

[18] Philippe Mestat. Lois de comportement des géomatériaux et modélisation par la méthode des éléments finis. Etudes et recherches des Laboratoires des Ponts et Chaussées - Serie Geotechnique, (GT 52), 1993.

[19] Pierre Humbert, Alain Dubouchet, Gérard Fezans, and David Remaud. CESAR-LCPC: A computation software package dedicated to civil engineering uses. Bulletin des laboratoires des ponts et chaussées, 256(257):7-37, 2005.

[20] Henar Herrero, Yvon Maday, and Francisco Pla. RB (Reduced basis) for RB (Rayleigh-Bénard). Computer Methods in Applied Mechanics and Engineering, 261:132-141, 2013.

[21] Olga Mula Hernandez. Quelques contributions vers la simulation parallèle de la cinétique neutronique et la prise en compte de données observées en temps réel. $\mathrm{PhD}$ thesis, Université Pierre et Marie Curie, 2014.

[22] F. Hecht. New development in freefem++. J. Numer. Math., 20(3-4):251-265, 2012. 